\title{
UTILIZAÇÃO DO MATERIAL DIDÁTICO KIT MOLA NO ENSINO REMOTO
}

DOI: 10.37702/2175-957X.COBENGE.2021.3611

Elisabeth Junges - bethjunges@gmail.com

Universidade Federal do Espírito Santo

Av. Dos Expedicionários, 11511151

29090-490 - Vitória - ES

Hiago Fernando Vagmaker Gonçalves - hiagofvg12@hotmail.com

Universidade Federal do Espírito Santo

Rua Elpídio Lima 16

29845-000 - Boa Esperança - ES

Resumo: Devido à nova realidade do ensino remoto, imposto pela pandemia do novo Corona vírus, os docentes vêm adaptando e buscando metodologias e ferramentas adequadas a esta modalidade de ensino. O objetivo deste texto é contribuir na adaptação e avaliação do material didático Kit Estrutural Mola no ensino remoto de estruturas. Amplamente utilizado no ensino presencial de estruturas, o Kit Mola permite a simulação de estruturas reais, auxiliando a análise e a compreensão de conceitos fundamentais sobre estruturas. Este material é empregado de forma adaptada no ensino remoto da disciplina de Análise Estrutural I, do curso de Engenharia Civil da Universidade Federal do Espírito Santo. São descritos os conteúdos produzidos e os exercícios propostos com esta ferramenta, tendo como principal objetivo a visualização pelos alunos dos conceitos estudados de forma teórica. De maneira a avaliar o impacto da utilização do Kit Mola no aprendizado, foi realizada uma pesquisa qualitativa com os discentes do semestre letivo corrente. Constatou-se que a utilização do Kit Mola no ensino remoto teve um impacto positivo no aprendizado. Ao final, lista-se algumas sugestões de aplicação deste material.

Palavras-chave: Ensino remoto. Kit Mola. Ferramenta didática. Estruturas. 


\section{UTILIZAÇÃO DO MATERIAL DIDÁTICO KIT MOLA NO ENSINO REMOTO}

\section{INTRODUÇÃO}

No processo de formação de um profissional, independente da sua área de atuação, garantir uma aprendizagem de qualidade é de suma importância para que no futuro o indivíduo possa exercer de forma adequada sua profissão. É papel das universidades garantirem a qualificação e aptidão do profissional, por meio da implantação de métodos de ensino que proporcionam experiências práticas abrangendo a tecnologia e a inovação (AGUIAR et al., 2016). Contudo, o ensino superior brasileiro historicamente se caracterizou por aulas expositivas como principal forma de aprendizagem. Em dias atuais, Colenci (2000) aponta que um modelo somente expositivo de ensino, já não é tão eficiente, uma vez que as habilidades essenciais que tal profissional necessitará, não são totalmente desenvolvidas.

Estendendo o problema citado anteriormente ao ensino de estruturas nos cursos de engenharia civil, Rebello (2000) sugere que sejam seguidas duas vertentes para adquirir as experiências necessárias sobre estruturas: a percepção e o conhecimento teórico de cálculo. $O$ autor ressalta que é essencial que tais conhecimentos teóricos e intuitivos sejam desenvolvidos em conjunto, para que o aluno não só aprenda a conceber uma estrutura, mas também a entenda. Essa percepção pode ser trabalhada, dentre outras maneiras, com a utilização de ferramentas que simulem com eficácia estruturas reais, auxiliando na melhor compreensão da funcionalidade de cada elemento que as compõem.

O Kit Estrutural Mola, criado pelo arquiteto Márcio Sequeira de Oliveira (OLIVEIRA, 2008), se apresenta com competência como uma ferramenta de simulação estrutural. Segundo Oliveira (2008, p. 155) o Mola Model "[...]mostrou-se bastante eficiente quando comparado a outros modelos estruturais qualitativos existentes, pois permite a avaliação do comportamento de diferentes estruturas sob diversos aspectos[...]". O produto, que é composto por imãs e peças modulares que se conectam permitindo a simulação de estruturas reais, vem sendo amplamente utilizado no ensino de estruturas, permitindo aos alunos a análise e a compreensão de conceitos fundamentais para esse tema, como a estabilidade estrutural e a deformação, compressão e tração dos componentes estruturais (IZIDORO; SILVA, 2018). Atualmente, além do Brasil, o Mola já é utilizado em outros 60 países ao redor do mundo (MOLAMODEL, 2020). A figura 1 mostra o material didático completo, que contém, além das peças para a simulação, um manual didático que aborda os principais conceitos que o kit permite trabalhar.

No formato de ensino presencial o Kit Mola já é amplamente difundido dentro das disciplinas de estruturas, se tornando uma ferramenta didática que contribui na qualidade da aprendizagem. Prova disso é a publicação de algumas pesquisas realizadas por docentes e discentes que utilizam o Mola Model como ferramenta de ensino e de pesquisa. Como exemplo, cita-se Izidoro e Silva (2018) que, utilizando o Kit Mola, avaliaram o entendimento de seus alunos de Engenharia sobre o comportamento estrutural de treliças. Os autores chegaram à conclusão que a utilização do Kit ajudou no aprimoramento da compreensão dos conceitos de tração e compressão dos alunos e serviu como reforço na compreensão do conceito de estabilidade.

A implantação do ensino remoto em muitas instituições de ensino, devido a pandemia do novo Corona vírus, trouxe uma situação nova e desafiadora para muitos docentes: a necessidade de adaptar o ensino presencial para o ensino remoto. Nesse 
processo de adaptação, busca-se garantir a qualidade do ensino e manter o nível de aprendizagem adequado. Assim, foi necessário, e ainda é, repensar as metodologias até então empregadas no ensino presencial, adaptando algumas para o ensino remoto, além de trazer novas estratégias e novos instrumentos para o ensino à distância.

Figura 1 - Kit Mola Model I.

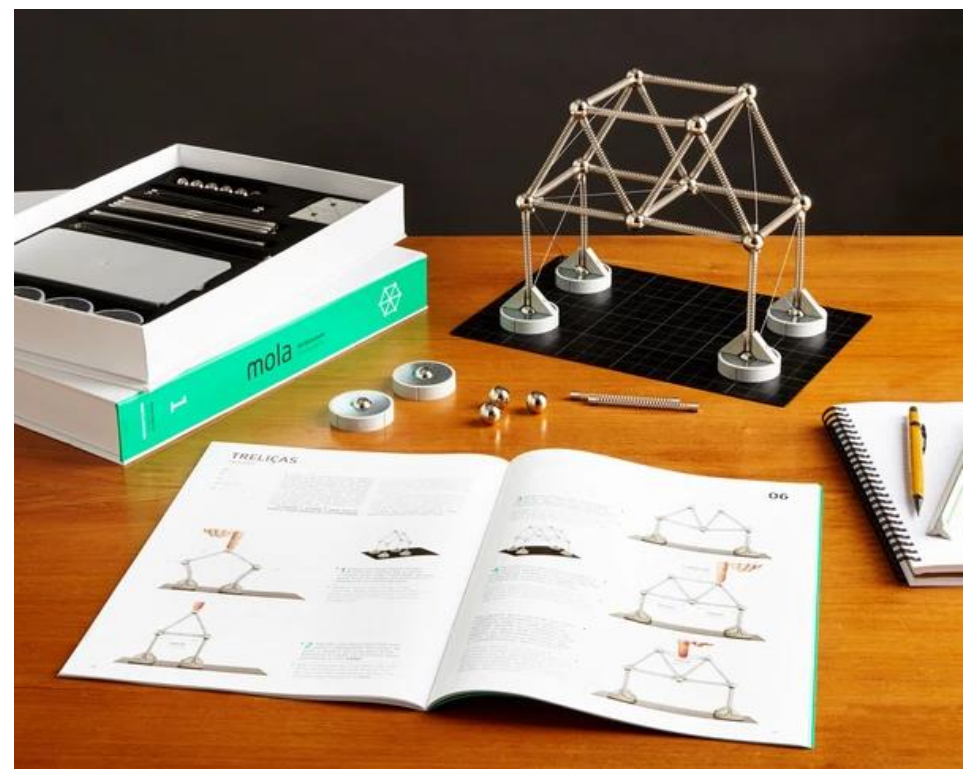

Fonte: Mola model (2020)

Nesse cenário, A Universidade Federal do Espírito Santo (UFES) implementou o ensino remoto emergencial (EARTE), que teve início em setembro de 2020, com o reinício do semestre letivo 2020/1. Até a finalização desde texto, o segundo semestre letivo nesta modalidade já estava em conclusão (semestre 2020/2), com previsão de haver no ano de 2021, no mínimo, mais um semestre nesta modalidade. Como ferramentas para implementação do ensino remoto adotam-se nesta Universidade as ferramentas do Google, como o Classroom e o Meet.

Com a perduração do formato remoto, percebe-se a necessidade de avaliar as metodologias de ensino empregadas, bem como incluir e testar novas ferramentas e estratégias de ensino. Diante de todo o exposto, quer-se, com este trabalho, compartilhar a metodologia de utilização da ferramenta didática Kit Estrutural Mola de forma remota na disciplina de Análise Estrutural I, do curso de Engenharia Civil da UFES. Neste texto são descritos os métodos de utilização do Kit Mola I e II em formato remoto. Adicionalmente, de maneira a avaliar o impacto de sua aplicação na aprendizagem dos alunos da disciplina, aplicou-se um formulário com questões simples, com o objetivo de realizar uma avaliação qualitativa.

\section{APLICAÇÃO DO KIT MOLA NO ENSINO REMOTO}

A disciplina de Análise Estrutural I faz parte das disciplinas obrigatórias e compõe a grade do terceiro período (ou semestre) do curso de Engenharia Civil. A ementa da disciplina aborda conteúdos sobre estática das estruturas, como: equações fundamentais da estática, estabilidade e estaticidade, diagramas de esforços internos de vigas, pórticos planos simples e compostos, arcos, grelhas, sendo todos isostáticos. Desta forma, o Kit Mola faz-se uma ferramenta visual excelente para ampliar a compreensão dos conceitos 
trabalhados, como: tipos de apoios, ligações rígidas e articuladas, estabilidade, efeitos dos esforços de tração/compressão e da flexão, etc. Assim, buscou-se desenvolver conteúdo com o Kit Mola que poderia ser aplicado no formato remoto.

A primeira aplicação proposta tem o objetivo de trazer parte da aula com o Kit Mola que a disciplina oferta na modalidade presencial. Desta forma, foi gravada uma videoaula com a utilização do kit mola I, em que são apresentados alguns conceitos básicos sobre estruturas, baseados no próprio manual do Mola. Alguns itens abordados: tipos de apoios; ligações internas; efeito da tração e compressão na barra; efeito da flexão; estabilidade de pórticos simples; o elemento de contraventamento. A figura 2 mostra dois momentos do vídeo disponibilizado no início do semestre aos alunos.

Figura 2 - Fotos extraídas da videoaula utilizando o kit mola I: a) apresentação da ligação rotulada (barra em balanço instável); b) avaliação da estabilidade de um pórtico plano.

a)

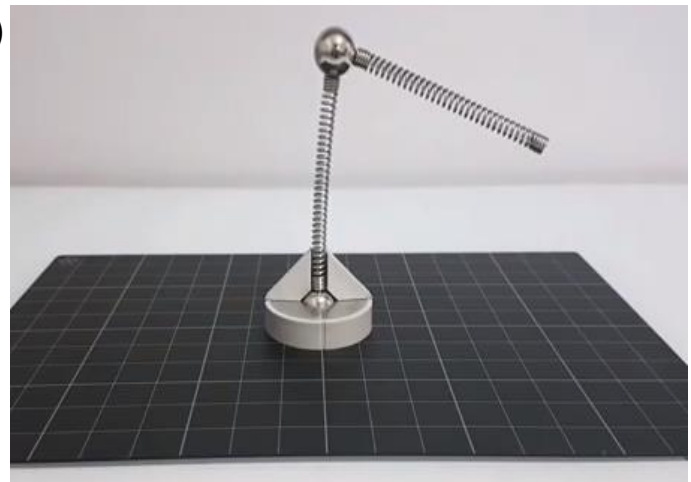

b)

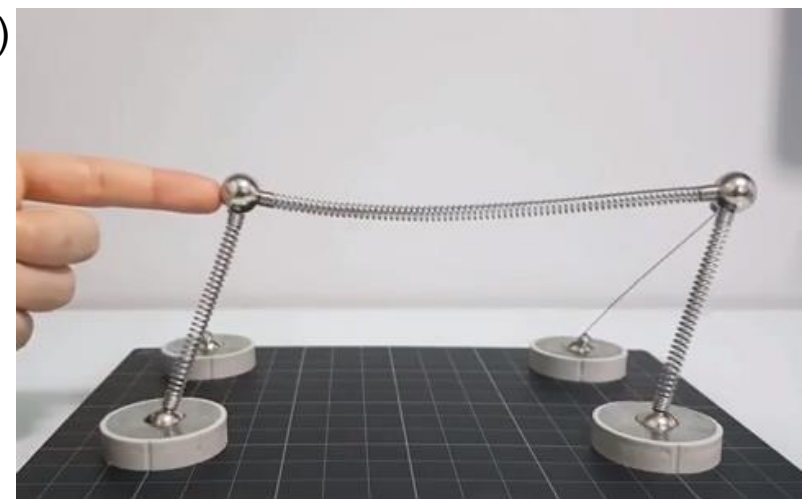

Fonte: Arquivo pessoal (2021)

Ao longo do semestre letivo, foram propostos alguns exercícios que continham maquetes de estruturas com o Mola Model, mostradas por meio de fotos. Os exercícios eram objetivos, disponibilizados aos alunos por meio de formulários do Google, em atividades dentro do ambiente da disciplina do Classroom. Na sequência, lista-se um resumo dos principais.

i. Exercício inicial - a partir da foto de uma maquete escolher, entre as alternativas de modelos teóricos disponíveis (desenho com indicação dos tipos de ligações internas e externas), a opção que corretamente representa a estrutura simulada.

ii. Exercício sobre estabilidade de pórtico plano - a partir da foto de uma maquete classificar a estrutura em estável ou instável, e classificá-la quanto a sua estaticidade em isostática, hiperestática ou hipostática (são dadas algumas alternativas).

iii. Exercício sobre diagrama de momento fletor e deformada de pórticos planos - em uma maquete de pórtico simples simula-se uma força concentrada e, para o caso de carregamento mostrado, pede-se que o aluno assinale a alternativa correspondente a deformada do pórtico e ao diagrama de momento fletor (Figura 3).

iv. Exercício de decomposição de pórtico composto - realizado durante aula assíncrona, mostram-se também fotos da maquete com kit mola (figura 4) de maneira a ajudar na visualização das diferentes partes que compõem e, ainda, ajudar o aluno a pensar na sequência de cálculo e de construção/montagem da estrutura. 


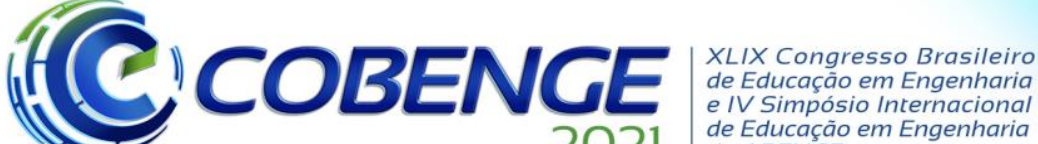

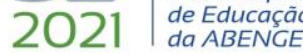 \\ 28 a 30 de SETEMBRO \\ Evento Online \\ "Formação em Engenharia: \\ Tecnologia, Inovação e Sustentabilidade"}

Figura 3 - Exercício proposto: a) pórtico triarticulado submetido a força horizontal; b) deformada correspondente; c) diagrama de momento fletor correspondente.

a)

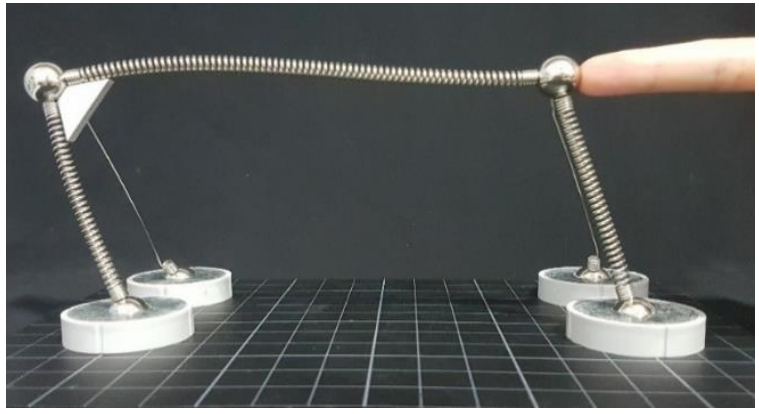

b)

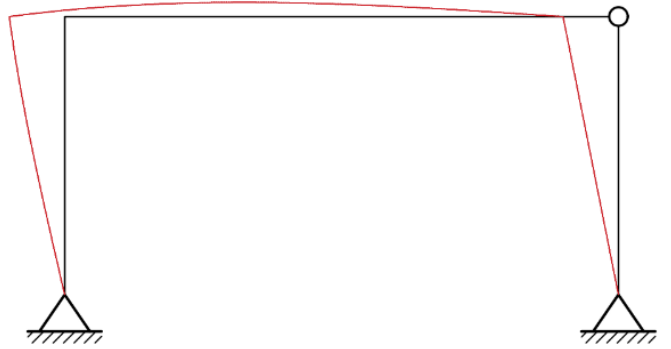

c)

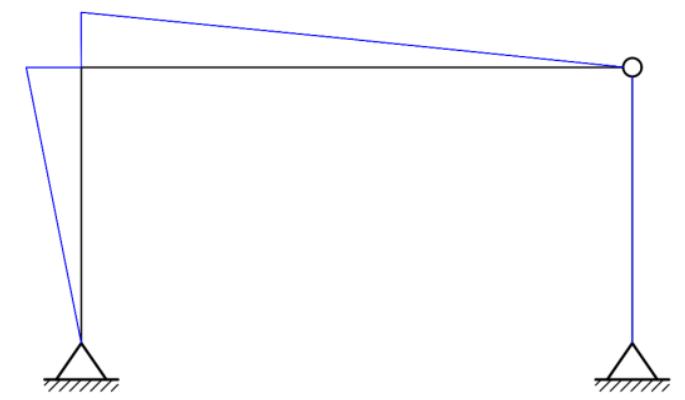

Fonte: Arquivo pessoal (2021).

Figura 4 - Exercício de decomposição de pórtico plano isostático composto.
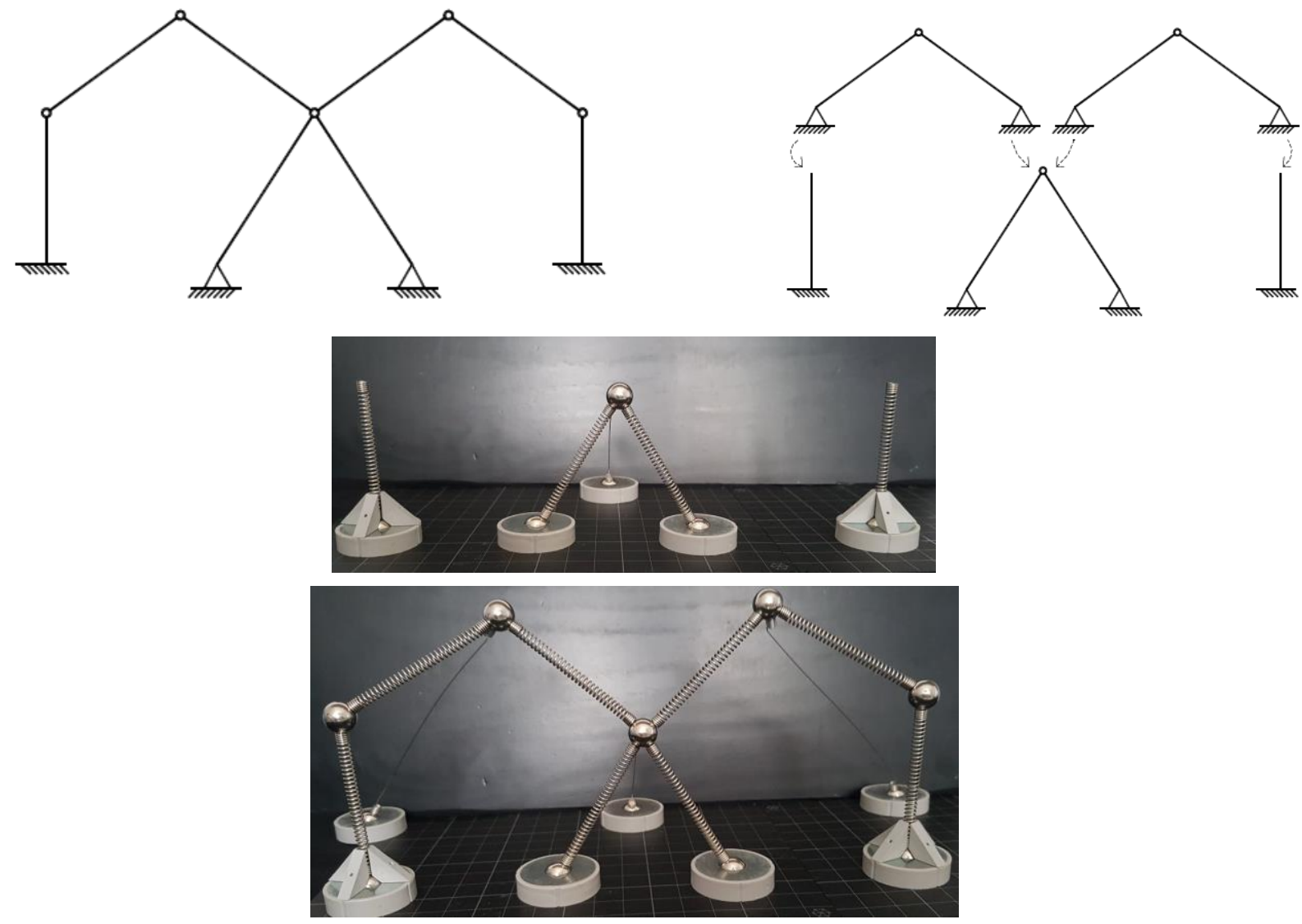

Fonte: Arquivo pessoal (2021). 
Além dos listados, empregou-se o Mola Model na simulação de maquete $3 \mathrm{~d}$ da estrutura aporticada a ser calculada pelos alunos em um trabalho assíncrono. Foram adicionados vídeos curtos para mostrar a instabilidade de um pórtico composto e para mostrar o funcionamento de grelhas. Enfim, ao longo do semestre letivo, pode-se observar a resposta dos alunos frente as diferentes atividades e a possibilidade de aplicação em diferentes elementos estruturais de diferentes formas.

\section{RESULTADOS}

Para avaliar a contribuição deste material didático no aprendizado remoto da disciplina de Análise Estrutural I, pela percepção dos próprios discentes, foi elaborado um formulário simples, com três questões, sendo duas objetivas e uma discursiva. O formulário foi aplicado por meio da plataforma Google Classroom e disponibilizado por uma semana, sendo que os 25 (vinte e cinco) discentes matriculados responderam as três questões. No momento de aplicação do formulário o semestre letivo encontrava-se em andamento, tendo vistos os seguintes tópicos principais: conceitos fundamentais (equações de equilíbrio, graus de liberdade, ligações externas e internas), equações fundamentais da estática, treliças planas, estabilidade e estaticidade, diagramas de esforços internos de vigas e pórticos planos simples e compostos. Este conteúdo compreende aproximadamente $80 \%$ (oitenta por cento) do conteúdo e carga horária da disciplina. Portanto, já era possível fazer uma avaliação qualitativa adequada da metodologia empregada.

As duas questões objetivas têm como finalidade avaliar a contribuição no aprendizado dos discentes sobre o conteúdo abordado na disciplina. A primeira questão se refere especificamente sobre a videoaula disponibilizada ao final do primeiro capítulo da disciplina: "Sobre o vídeo apresentado com o kit mola I, em que foram revisados e explicados conceitos básicos de estruturas. Qual o nível de importância dele no seu entendimento sobre os conceitos abordados (vistos antes do vídeo de forma teórica/exercícios numéricos), como: tipos de apoios, graus de liberdade, ligação rígida e ligação rotulada?".

As respostas obtidas estão mostradas na Figura 5. Todos os alunos confirmaram a contribuição positiva (10 alunos) ou extremamente positiva (15 alunos).

Figura 5 - Resultado da primeira questão.

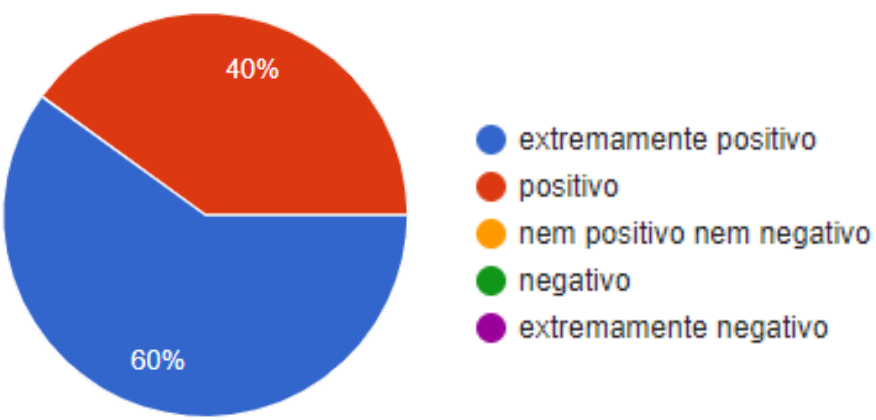

Fonte: Arquivo Pessoal (2021).

A segunda questão se refere aos exercícios e demais aplicações ao longo da disciplina. Foram dadas as mesmas opções de resposta da primeira questão. A segunda pergunta era a seguinte: "Sobre os exercícios com maquetes de estruturas utilizando o kit mola no entendimento do conteúdo. Aqui entram: (1) a atividade sobre kit mola feito mais 
no início da disciplina; (2) a maquete do trabalho; (3) os exercícios de decomposição e estabilidade de pórticos compostos; (4) atividade sobre diagramas e deslocamentos de pórticos. Avalie o emprego do kit mola nestes exercícios".

Figura 6 - Resultado da segunda questão do formulário

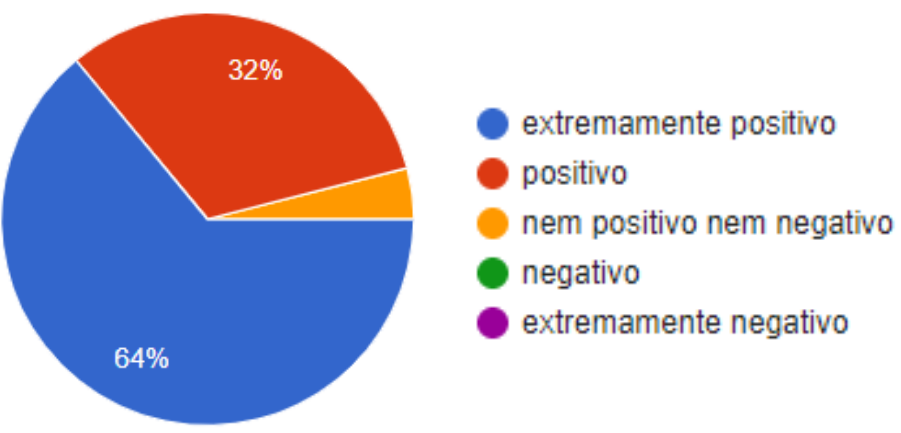

Fonte: Arquivo Pessoal (2021).

Na segunda questão o retorno também foi positivo. Como mostra a figura 6, 8 (oito) discentes responderam como positivo e 16 (dezesseis) como extremamente positivo o emprego do kit mola em atividades remotas, apenas uma resposta (4\%) foi "nem positivo nem negativo".

Para concluir, a terceira questão foi aberta com o objetivo de obter sugestões gerais sobre a aplicação do kit mola nas aulas em formato remoto, tendo assim, uma visão dos próprios estudantes. A pergunta colocada no formulário foi a seguinte: "De acordo com sua experiência até agora sobre a utilização de maneira remota do material didático kit mola, comente se gostaria de ver mais aplicações na disciplina, faça sugestões de que outras maneiras gostariam de ver aplicado este material dentro do conteúdo, enfim, comente de forma livre". Das 25 (vinte e cinco) respostas obtidas, a maioria sugeria a ampliação da aplicação deste material nas aulas, principalmente em exercícios. Outro ponto mais citado é a ajuda do kit mola (por fotos e vídeos) no entendimento da decomposição de pórticos compostos e na avaliação da estabilidade, pedindo que seja mais utilizado.

Diante dos comentários colocados pelos discentes, junto também com o resultado dos exercícios propostos e percepções gerais ao longo da disciplina, pode-se destacar alguns pontos sobre a aplicação do kit mola em atividades remotas, listados na sequência.

i. Os exercícios propostos normalmente envolvem a representação teórica da estrutura que está simulada em kit mola. De maneira que o aluno assimile mais facilmente o modelo físico ao modelo teórico, os alunos demonstraram a necessidade de aplicação de mais exercícios iniciais, em que o objetivo é idealizar o modelo teórico a partir do modelo do kit mola. Assim, iniciar a aplicação do kit mola no ensino remoto por meio desse exercício, garantirá melhor aproveitamento e desempenho do aluno em atividades sequentes;

ii. Para atividades de avaliação da estabilidade de estruturas aporticadas, a adição de vídeos curtos, principalmente nos exemplos instáveis onde a maquete submetida a uma estimulação (força aplicada pela mão) desaba, mostrou-se ser uma ferramenta eficaz para auxiliar no entendimento deste tópico. Vários alunos sugeriram o aumento da utilização do kit mola em exercícios de estabilidade, dando preferência aos vídeos.

iii. Apesar de não ser o foco da disciplina o estudo dos deslocamentos (configuração deformada) provocados pelas cargas externas aplicadas, os alunos mostraram 
interesse e comentaram que tal informação auxilia no entendimento do comportamento estrutural e, consequentemente, na assimilação dos diagramas de esforços internos (especificamente, trabalhou-se nos exercícios com o diagrama de momento fletor de pórticos simples), que é o assunto central da disciplina.

\section{CONSIDERAÇÕES FINAIS}

O emprego de diferentes metodologias e materiais didáticos adaptados ao ensino remoto é essencial para garantir a qualidade da aprendizagem nesta modalidade. Como o ensino de estruturas nos cursos de Engenharia Civil sempre foi predominantemente em formato presencial, ainda se avalia quais as ferramentas e metodologias podem ter impacto positivo no aprendizado. Portanto, é importante avaliar a eficiência de tais instrumentos.

O material didático Mola Model, apesar de ter sido desenvolvido para o aprendizado de estruturas por meio do contato direto com a ferramenta, que é o ideal, mostra-se eficiente para o ensino remoto. Por meio da pesquisa qualitativa realizada com os discentes da disciplina de Análise Estrutural I do curso de Engenharia Civil da UFES, semestre letivo 2020/2, pode-se fazer uma avaliação qualitativa inicial do impacto desta ferramenta no ensino à distância. Pelas respostas dos discentes, obteve-se um retorno totalmente positivo e ainda algumas sugestões. Destaca-se a necessidade de realizar exercícios iniciais para a assimilação do modelo teórico estrutural que a maquete representa. Adicionalmente, constata-se a importância dos vídeos para introdução dos conceitos básicos que envolvem a estática das estruturas, como: tipos de apoios, ligações internas, efeito do esforço normal (tração e compressão), efeito da flexão. O destaque da utilização deste material didático de forma remota é a melhor visualização dos alunos em problemas de estabilidade de estruturas aporticadas. Enfatiza-se, ainda, as possibilidades de ampliação de sua utilização, como por exemplo, por meio de mais vídeos curtos e abordagem dos outros elementos de barras estudados, como as treliças e as grelhas.

\section{Agradecimentos}

Ao Laboratório Didático de Estruturas - LabDEst, pertencente ao Departamento de Engenharia Civil da UFES, pelo fornecimento do material Kit Mola I e Kit Mola II. À UFES pela bolsa de Iniciação Científica do segundo autor.

\section{REFERÊNCIAS}

AGUIAR, Ana Luiza S. de et al. A importância das atividades práticas no ensino de instalações elétricas prediais - um estudo de caso. In: XLIV CONGRESSO BRASILEIRO DE EDUCAÇÃO EM ENGENHARIA, 2016, Natal. Anais. Macaé. Disponível em: http://www.abenge.org.br/cobenge/legado/arquivos/3/anais/anais/155799.pdf. Acesso em: 17 mai.2020.

COLENCI, Ana Teresa. 0 ensino de engenharia como uma atividade de serviços: a exigência de atuação em novos patamares de qualidade acadêmica. 2000. $130 \mathrm{f}$. Dissertação (Mestrado) - Curso de Engenharia de Produção, Universidade de São Paulo, São Carlos, 2000. Disponível em: https://www.teses.usp.br/teses/disponiveis/18/18140/tde-14052004150657/publico/Dissertacao Ana Teresa Colenci2.pdf. Acesso em: 15 mai.2020. 
IZIDORO, Diego Luís; SILVA, Danielli Ferreira. Análise de treliças utilizando o modelo estrutural qualitativo mola. In: XLVI CONGRESSO BRASILEIRO DE EDUCAÇÃO EM ENGENHARIA, 2018, Salvador. Anais. Formiga, 2018. Disponível em:

http://www.abenge.org.br/sis artigos.php. Acesso em: 17 mai.2020.

REBELLO, Yopanan Conrado Pereira. A concepção estrutural e a arquitetura. São Paulo: Zigurate Editora, 2000.

MOLAMODEL. MOLA STRUCTURAL KIT: uma nova maneira de estudar e ensinar estruturas. 2020. Disponível em: https://molamodel.com/. Acesso em: 21 mai.2020.

OLIVEIRA, Márcio Sequeira de. Modelo Estrutural Qualitativo Para Pré-avaliação do Comportamento de Estruturas Metálicas. 2008. 187 f. Dissertação (Mestrado) - Curso de Programa de Pós-graduação em Engenharia Civil, Universidade de Ouro Preto - Escola de Minas, Ouro Preto, 2008. Disponível em:

https://www.propec.ufop.br/teses-e-dissertacoes/148/modelo-estrutural-qualitativo-parapre-avaliacao-do-comportamento-de-estruturas-metalicas. Acesso em: 21 mai.2020.

\section{USE OF MOLA STRUCTURAL KIT IN DISTANCE LEARNING}

Abstract: Due to the new reality of distance learning, imposed by pandemic of the new coronavirus, educators have been adapting and seeking methodologies and tools appropriate to this type of teaching. The purpose of this text is to contribute to the adaptation and evaluation of the material Mola Structural Kit in the distance learning of structures. Widely used in classroom teaching of structures, Mola allows the simulation of real structures, assisting in the analysis and understanding of fundamental concepts about structures. This material is used in an adapted way in the online learning of Structural Analysis I, from the Civil Engineering course at the Federal University of Espirito Santo. The contents produced and the exercises proposed with this tool are described, having as main objective the visualization of the theoretical concepts. To assess the impact of using the Mola kit, a qualitative research was carried out with the undergraduates' students of the current academic semester. It was found that the use of Mola in online learning had a positive impact on learning. At the end, it is listed some suggestions for applying this material.

Keywords: Distance learning. Mola Kit. Educational tool. Structures. 